\title{
ACADEMY OF SCIENCES OF THE CZECH REPUBLIC INSTITUTE of MATHEMATICS
}

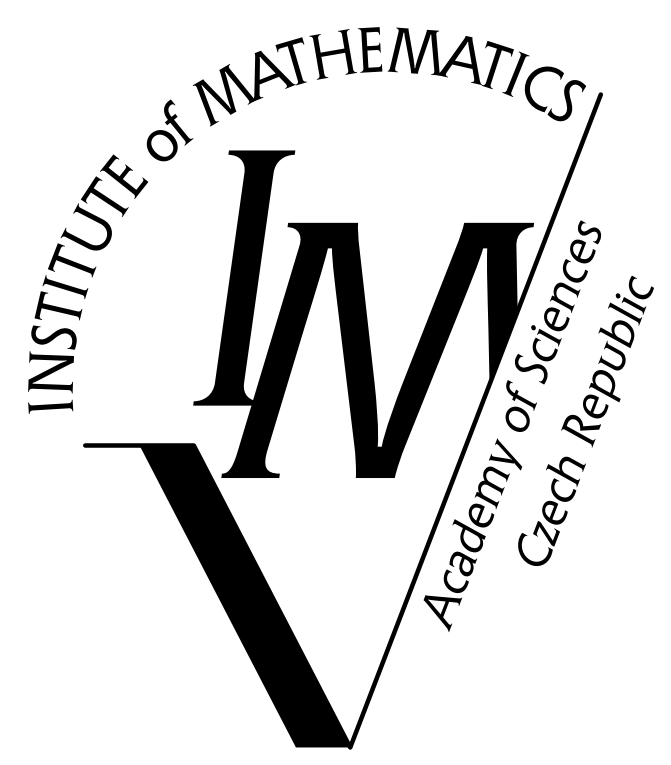

ON OPTIMALITY CONDITIONS IN CONTROL OF ELLIPTIC VARIATIONAL INEQUALITIES

Jiři Outrata, Jiři Jarušek, Jana Stará

(preprint)

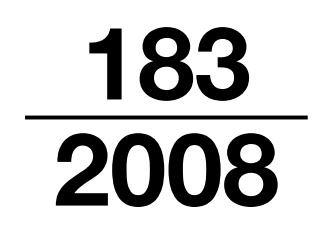




\title{
On optimality conditions in control of elliptic variational inequalities *
}

\author{
Jiří Outratał Jiří Jarušek jana Stará ${ }^{\S}$
}

\begin{abstract}
In the paper we consider optimal control of a class of strongly monotone variational inequalities, whose solution map is directionally differentiable in the control variable. This property is used to derive sharp pointwise necessary optimality conditions provided we do not impose any control or state constraints. In presence of such constraints we make use of the generalized differential calculus and derive, under a mild constraint qualification, optimality conditions in a "fuzzy" form. For strings, these conditions may serve as an intermediate step toward pointwise conditions of limiting (Mordukhovich) type. For membranes, however, limiting conditions cannot be derived in this way.
\end{abstract}

Keywords. Directional differentiability, critical cone, strong local fuzzy sum rule, calmness, capacity.

\section{Introduction}

Numerous important optimization problems arising in continuum mechanics, economy, transportation networks etc. can be modeled as optimal control of variational inequalities or complementarity problems. Since 1996 (cf. [10]) these models are considered in the framework of mathematical programs with equilibrium constraints (MPECs). Early works on this subject arose, however, already in the seventies (cf. [4],[11]) and the development has proceeded all the time.

In a recent monograph, [12, Chapter 5], Mordukhovich has applied advanced tools of variational analysis and generalized differential calculus to derive necessary optimality conditions for a class of infinite-dimensional MPECs in which the equilibria are governed by various types of generalized equations (GEs). These conditions require besides the standard constraint qualifications (CQs) also the so-called sequential-normal-compactness (SNC) conditions which ensure a certain minimal amount of compactness needed to apply the basic rules of generalized differentiation in infinite dimensions. In some special situations under a surjectivity assumption none of the above conditions are needed, but the derivation of workable optimality conditions of this type still remains a difficult task. The reason consists

*The work presented here was partially supported by the Czech Academy of Sciences under grant IAA 100750802 and under the Institutional research plan AVOZ 10190503. The third author was supported by the Czech Grant Agency under the grant GACR 201/06/0352 and by the research project MSM0021620839.

${ }^{\dagger}$ Institute of Information Theory and Automation, Acad. Sci. of the Czech Republic, Pod vodárenskou věží 4, 18208 Praha 8, Czech Republic (outrata@utia.cas.cz).

¥Mathematical Institute, Academy of Sciences of the Czech Republic, Žitná 25, 11567 Praha 1, Czech Republic (jarusek@math.cas.cz).

$\S$ Dpt. of Mathematical Analysis, Faculty of Mathematics and Physics, Charles University, Sokolovská 83, 18600 Praha 8, Czech Republic (stara@karlin.mff.cuni.cz). 
in our inability to express weak* limits of sequences in some functions spaces in a suitable form preserving the sharpness (selectivity) of the resulting optimality conditions. This hurdle has been successfully taken in [8] in the special case of control of a contact problem with a string. The main argument came there from the compact embedding of $\stackrel{\circ}{H}^{1}(0,1)$ into $C^{0}(0,1)$, which has enabled to derive sharp pointwise conditions, very close to the corresponding finite-dimensional case. Unfortunately, this argument cannot be used whenever we replace an interval by a two-dimensional domain. (Observe that a higher dimension has no physical meaning).

The aim of this paper is threefold:

(i) To derive for a class of infinite-dimensional MPECs including the problem from [8] sharp "fuzzy" optimality conditions with a weak (nonrestrictive) CQ;

(ii) to use the obtained fuzzy conditions as an intermediate step on the way to conditions in terms of limiting objects for the MPEC from [8];

(iii) to analyse the bounds of the approach (ii) in the presented function-space setting.

The organization of the paper is as follows. In Section 2 our MPEC is formulated and a crucial auxiliary assertion is stated. Section 3 is devoted to the special case without any control or state constraints. In this situation our approach directly leads to very sharp pointwise conditions corresponding to the notion of strong stationarity from [15]. In Section 4 the fuzzy optimality conditions are derived under a weak calmness CQ that is automatically fulfilled whenever one has to do with control constraints only. These conditions enable us to recover the result from [8] in a different way. The last Section 5 shows then by means of a sophisticated example that the proof techniques from [8] or from Section 4 cannot be directly extended beyond the one-dimensional domains.

Our notation is standard: for a closed set $A, \delta_{A}(\cdot)$ stands for the indicatory function of $A, d_{A}(\cdot)$ is the distance to $A$ and $T_{A}(\bar{x})$ denotes the Bouligand (contingent) cone of $A$ at $\bar{x} \in A$. For a cone $D$ with vertex at $0, D^{\circ}$ denotes its negative polar. $\mathbb{B}(x, r), \overline{\mathbb{B}}(x, r)$ stand for the open and closed ball centered at $x$ with radius $\mathrm{r}$, respectively; $\mathbb{B}:=\mathbb{B}(0,1)$ and $\bar{\Omega}$ is the closure of $\Omega$. For a mapping $F$, Gr $F$ denotes its graph.

For the readers' convenience we state now the definitions of several basic notions from modern variational analysis. For a detailed description of their properties, the reader is referred to the monographs [14] and [12].

Given a closed set $A$ in an Asplund space $X$ and a point $\bar{x} \in A$, we denote by $\widehat{N}_{A}(\bar{x})$ the Fréchet (regular) normal cone to $A$ at $\bar{x}$, defined by

$$
\widehat{N}_{A}(\bar{x})=\left\{x^{*} \in \mathbb{R}^{n} ; \limsup _{x \underset{A}{\longrightarrow} \bar{x}} \frac{\left\langle x^{*}, x-\bar{x}\right\rangle}{\|x-\bar{x}\|} \leq 0\right\} .
$$

The limiting (Mordukhovich) normal cone to $A$ at $\bar{x}$, denoted $N_{A}(\bar{x})$, is defined by

$$
N_{A}(\bar{x}):=\operatorname{Limsup}_{x \stackrel{A}{\longrightarrow} \bar{x}} \widehat{N}_{A}(x),
$$

where "Limsup" is the Kuratowski-Painlevé outer limit of sets (see [12]). If $A$ is convex, then $N_{A}(\bar{x})=\widehat{N}_{A}(\bar{x})$ amounts to the classical normal cone in the sense of convex analysis. We say that $A$ is normally regular at $\bar{x}$, provided $N_{A}(\bar{x})=\widehat{N}_{A}(\bar{x})$. 
For an extended-real-valued function $f[X \rightarrow \overline{\mathbb{R}}]$ and $\bar{x} \in \operatorname{dom} f$

$$
\widehat{\partial} f(\bar{x})=\left\{\xi \in X^{*} ;(\xi,-1) \in \widehat{N}_{\text {epi } f}(\bar{x}, f(\bar{x}))\right\}
$$

is the Fréchet subdifferential of $f$ at $\bar{x}$. For an indicatory functional $\delta_{A}$ one has $\widehat{\partial} \delta_{A}(\bar{x})=$ $\widehat{N}_{A}(\bar{x})$.

\section{Problem formulation and preliminaries}

Throughout the paper, we are dealing with the MPEC

$$
\begin{array}{ll}
\operatorname{minimize} \varphi(x, y) & \\
\text { subject to } & \\
& 0 \in \mathscr{A} y-x+a+N_{D}(y) \\
& x \in \omega \\
& y \in \Xi,
\end{array}
$$

where $x, a \in H^{-1}(\Omega), y \in \stackrel{\circ}{H}^{1}(\Omega), \mathscr{A}\left[\stackrel{\circ}{H}^{1}(\Omega) \rightarrow H^{-1}(\Omega)\right]$ is linear and elliptic, $D=\{v \in$ $\stackrel{\circ}{H}^{1}(\Omega) ; v(s) \geq 0$ a.e. in $\left.\Omega\right\}$, the sets $\omega \subset H^{-1}(\Omega), \Xi \subset \stackrel{\circ}{H}^{1}(\Omega)$ are nonempty and closed, and $\varphi\left[H^{-1}(\Omega) \times \stackrel{\circ}{H}^{1}(\Omega) \rightarrow \mathbb{R}\right]$ is continuously differentiable. It is well-known and easily verifiable that under these assumptions the solution map

$$
S(x):=\left\{y \in \stackrel{\circ}{H}^{1}(\Omega) ; 0 \in \mathscr{A} y-x+a+N_{D}(y)\right\}
$$

is single-valued and Lipschitz. In this way, (2.1) can be considered as a special optimal control problem and therefore we will entitle the variables $x$ and $y$ as control and state variable, respectively.

In the sequel, we consider sets where a function $y \in \stackrel{\circ}{H}^{1}$ attains either positive or zero values. In the framework of the classical definition of $\stackrel{H}{H}^{1}(\Omega)$ these sets are defined up to a set of Lebesgue measure zero which is too coarse for our case. Hence we will use capacity [see [2], Section 6.4.3] to get more precise results. We start with some elements of capacity theory.

Definition 2.1. ([2, Definition 6.47]) Let $A$ be a Borel subset of $\Omega$ and $\alpha \in \mathbb{R}$.

(i) We say that $y \in \stackrel{\circ}{H}^{1}(\Omega)$ satisfies the inequality $y \geq \alpha$ over $A$ in the sense of $\stackrel{\circ}{H}^{1}(\Omega)$ if there exists a sequence $y_{n} \rightarrow y$ in $\stackrel{\circ}{H}^{1}(\Omega)$ such that $y_{n} \geq \alpha$ over a neighborhood of $A$.

(ii) The capacity of $A$ (in the sense of $\stackrel{\circ}{H}^{1}(\Omega)$ ) is defined as ${ }^{1}$

$$
\operatorname{cap}(A):=\inf \left\{\|y\|^{2} ; y \geq 1 \text { over } A\right\} .
$$

(iii) We say that a measurable function $y$ is quasi-continuous if there is a non-increasing sequence $\Omega_{n}$ of open subsets of $\Omega$ such that $f$ is continuous on $\Omega \backslash \Omega_{n}$ and $\operatorname{cap}\left(\Omega_{n}\right) \rightarrow 0$.

(iv) We say that a property $P$ of $y \in \stackrel{\circ}{H}^{1}(\Omega)$ holds quasi everywhere (q.e.) if there exists a subset $E$ of $\Omega$ so that $P$ holds on $\Omega \backslash E$ and $\operatorname{cap}(E)=0$.

It is obvious that $\operatorname{cap}(A)$ is greater or equal to the Lebesgue measure of $A$. Denote by $M_{+}(\Omega)$ (resp. $M_{-}(\Omega)$ ) the set of all nonnegative (resp. nonpositive) Radon measures on $\Omega$, and by $\left(H^{-1}(\Omega)\right)_{+}$the positive cone in $\left(H^{-1}(\Omega)\right)$.

\footnotetext{
${ }^{1}$ By $\|y\|$ we understand the norm of $y$ in $\stackrel{\circ}{H}^{1}(\Omega)$.
} 
Lemma 2.2. The capacity is a nonnegative, subaditive set function having the following properties:

(i) For $y \in \stackrel{\circ}{H}^{1}(\Omega)$ there is a quasi-continuous everywhere defined function $\widetilde{y}$ such that $y(s)=\widetilde{y}(s)$ q.e.on $\Omega$. Thus $\widetilde{y}$ belongs to the equivalence class of $y$. (See [2, Lemma 6.50].)

(ii) $H^{-1}(\Omega)_{+}=H^{-1}(\Omega) \cap M_{+}(\Omega)$.(See [2, Theorem 6.54] and its consequences.)

(iii) Let $A$ be a Borel set. Then $A$ has null capacity if and only if $\mu(A)=0$ for every $\mu \in H^{-1}(\Omega) \cap M_{+}(\Omega)$.(See [2, Lemma 6.55].)

(iv) Let $y \in \stackrel{\circ}{H}^{1}(\Omega), \mu \in H^{-1}(\Omega) \cap M_{+}(\Omega)$. Then $y \in L_{1}(\Omega ; \mu)$ and

$$
<\mu, y>_{H^{-1}(\Omega), \stackrel{H}{H}^{1}(\Omega)}=\int_{\Omega} y d \mu .
$$

(See [2, Lemma 6.56].)

So, when speaking about $y \in \stackrel{H}{H}^{1}(\Omega)$, we will always consider its quasi continuous representative. Let us associate with each $y \in D$ the sets

$$
\begin{aligned}
L(y) & :=\{s \in \Omega ; y(s)>0\} \\
I(y) & :=\{s \in \Omega ; y(s)=0\} \\
K(y) & :=\Omega \backslash(L(y) \cup I(y)) .
\end{aligned}
$$

According to [2, Lemma 6.49], $\operatorname{cap}(K(y))=0$ and $y \geq 0$ q.e. on $\Omega$. Further, by [2, Theorem $6.57]$

$$
T_{D}(y)=\left\{z \in \stackrel{\circ}{H}^{1}(\Omega) ; z \geq 0 \text { q.e. on } I(y)\right\}
$$

and

$$
\widehat{N}_{D}(y)=\left\{\mu \in H^{-1}(\Omega) \cap M_{-}(\Omega) ; \mu(L(y))=0\right\} .
$$

In the sequel, we will also make use of [11], according to which $S$ is Hadamard differentiable at any $x$ in the direction $d$. To evaluate this directional derivative, one has

$$
S^{\prime}(x ; d)=v,
$$

where $v$ is the unique solution of the GE

$$
0 \in \mathscr{A} v-d+N_{C(x)}(v)
$$

with the critical cone

$$
\begin{aligned}
C(x) & =T_{D}(y) \cap(\mu)^{\perp}=\left\{z \in \stackrel{\circ}{H}^{1}(\Omega) ; z \geq 0 \text { q.e. on } I(y),\langle\mu, z\rangle=0\right\}, \\
\mu & =-\mathscr{A} y+x-a .
\end{aligned}
$$

In addition to the sets (2.2) let us introduce the sets:

$$
\begin{aligned}
I_{+}(\mu) & :=\operatorname{supp} \mu \\
I_{0}(y, \mu) & :=I(y) \backslash I_{+}(\mu)
\end{aligned}
$$

The arguments at $L, I, I_{+}$and $I_{0}$ will be omitted whenever this cannot cause any confusion. In terms of these sets

$$
C(x) \subset\left\{z \in \stackrel{\circ}{H}^{1}(\Omega) ; z \geq 0 \text { q.e. on } I_{0}(y, \mu), z=0 \mu \text {-a.e. on } I_{+}(\mu)\right\} \text {. }
$$




\section{Unconstrained case}

Consider a reference pair $(\bar{x}, \bar{y}) \in \operatorname{Gr} S$ and put

$$
\bar{\mu}=-\mathscr{A} \bar{y}+\bar{x}-a\left(\text { i.e. } \bar{\mu} \in N_{D}(\bar{y})\right) \text {. }
$$

It is clear from the Hadamard differentiability of $S$ that

$$
T_{\mathrm{Gr} S}(\bar{x}, \bar{y})=\left\{(d, v) \in H^{-1}(\Omega) \times \stackrel{\circ}{H}^{1}(\Omega) ; 0 \in \mathscr{A} v-d+N_{C(\bar{x})}(v)\right\},
$$

where $C(\bar{x})$ is given by $(2.6)$ with $x, y, \mu$ replaced by $\bar{x}, \bar{y}, \bar{\mu}$, respectively. Let $\widetilde{N}$ denote the negative polar of $T_{\mathrm{Gr} S}(\bar{x}, \bar{y})$.

\section{Lemma 3.1.}

$$
\widetilde{N}=\left\{(p, q) \in \stackrel{\circ}{H}^{1}(\Omega) \times H^{-1}(\Omega) ; \mathscr{A}^{*} p+q \in(C(\bar{x}))^{\circ}, p \in C(\bar{x})\right\} .
$$

Proof. By definition,

$$
\begin{aligned}
\widetilde{N} & =\left\{(p, q) ;\langle p, d\rangle+\langle q, v\rangle \leq 0 \forall(d, v) \in T_{\mathrm{Gr} S}(\bar{x}, \bar{y})\right\} \\
& =\left\{(p, q) ;\langle p, \mathscr{A} v+\xi\rangle+\langle q, v\rangle \leq 0 \forall(v, \xi) \text { such that } \xi \in N_{C(\bar{x})}(v)\right\} \\
& =\left\{(p, q) ;\left\langle\mathscr{A}^{*} p+q, v\right\rangle+\langle p, \xi\rangle \leq 0 \forall(v, \xi) \in \operatorname{Gr} N_{C(\bar{x})}\right\} .
\end{aligned}
$$

Since $C(\bar{x})$ is a closed convex cone, one has

$$
\operatorname{Gr} N_{C(\bar{x})}=\left\{(v, \xi) \in \stackrel{\circ}{H}^{1}(\Omega) \times H^{-1}(\Omega) ; v \in C(\bar{x}), \xi \in(C(\bar{x}))^{\circ},\langle\xi, v\rangle=0\right\} .
$$

If we ignore the complementarity condition in (3.3), we get a set, say $Q$, not smaller than $\operatorname{Gr} N_{C}(\bar{x})$. Consequently,

$$
\begin{aligned}
\widetilde{N} & \supset\left\{(p, q) ;\left\langle\mathscr{A}^{*} p+q, v\right\rangle+\langle p, \xi\rangle \leq 0 \forall(v, \xi) \in Q\right\} \\
& \supset\left\{(p, q) ; \mathscr{A}^{*} p+q \in(C(\bar{x}))^{\circ}, p \in C(\bar{x})\right\} .
\end{aligned}
$$

On the other hand, if we set first $v=0$ and then $\xi=0$, we obtain the opposite inclusion and the claim holds.

Our next task is to find a suitable description of $(C(\bar{x}))^{\circ}$.

Lemma 3.2. Let $\eta \in(C(\bar{x}))^{\circ}$. Then one has

(i) $\langle\eta, z\rangle=0$ for all $z \in \stackrel{\circ}{H}^{1}(\Omega)$ such that $z=0$ q.e. on $I(\bar{y})$;

(ii) $\langle\eta, z\rangle \leq 0$ for all $z \in D$ such that $\langle\bar{\mu}, z\rangle=0$.

Proof. Select a test function $z \in \stackrel{\circ}{H}^{1}(\Omega)$ satisfying the condition $z=0$ q.e. on $I(\bar{y})$. Since $\bar{\mu}(L(\bar{y}))=0$, one has then $\langle\bar{\mu}, z\rangle=0$, and so $\pm z \in C(\bar{x})$. This implies condition (i).

(ii) follows directly from (2.6).

Remark 3.3. A weaker version of (i) attains the form

$$
\langle\eta, z\rangle=0 \text { for all } z \in \stackrel{\circ}{H}^{1}(\Omega) \text { such that } \bar{y} \pm \varepsilon z \in D \text { for some } \varepsilon>0 \text {. }
$$


On the basis of the above lemmas one can immediately derive sharp optimality conditions for the unrestricted case when $\omega=H^{-1}(\Omega)$ and $\Xi=\stackrel{\circ}{H}^{1}(\Omega)$.

Theorem 3.4. Let $(\widehat{x}, \widehat{y})$ be a (local) solution of the MPEC

$$
\begin{array}{ll}
\text { minimize } & \varphi(x, y) \\
\text { subject to } & \\
& 0 \in \mathscr{A} y-x+a+N_{D}(y) .
\end{array}
$$

Then there exist multipliers $\widehat{p} \in \stackrel{\circ}{H}^{1}(\Omega), \widehat{\eta} \in H^{-1}(\Omega)$ such that

$$
\begin{aligned}
& 0=\nabla_{x} \varphi(\widehat{x}, \widehat{y})+\widehat{p} \\
& 0=\nabla_{y} \varphi(\widehat{x}, \widehat{y})-\mathscr{A}^{*} \widehat{p}+\widehat{\eta}
\end{aligned}
$$

and, additionally, with $\widehat{\mu}=-\mathscr{A} \widehat{y}+\widehat{x}-a$, they fulfill the conditions

(i) $\widehat{p} \geq 0$ q.e. on $I_{0}(\bar{y}, \bar{\mu})$;

(ii) $\widehat{p}=0$ $\widehat{\mu}$-a.e.on $I_{+}(\bar{\mu})$;

(iii) $\langle\widehat{\eta}, z\rangle=0$ for all $z \in \stackrel{\circ}{H}^{1}(\Omega)$ such that $z=0$ q.e. on $I(\bar{y})$;

(iv) $\langle\widehat{\eta}, z\rangle \leq 0$ for all $z \in D$ such that $\langle\widehat{\mu}, z\rangle=0$.

Proof. By virtue of continuous differentiability of $\varphi$, one has

$$
0 \in \nabla \varphi(\widehat{x}, \widehat{y})+\widehat{N}_{\mathrm{Gr} S}(\widehat{x}, \widehat{y}) .
$$

As shown eg in [12, Cor. 1.11],

$$
\widehat{N}_{\mathrm{Gr} S}(\widehat{x}, \widehat{y}) \subset\left(T_{\mathrm{Gr} S}(\widehat{x}, \widehat{y})\right)^{\circ}=\widetilde{N}
$$

Consequently, by virtue of [1],

$$
\begin{aligned}
& 0=\nabla_{x} \varphi(\widehat{x}, \widehat{y})+\widehat{p} \\
& 0=\nabla_{y} \varphi(\widehat{x}, \widehat{y})-\mathscr{A}^{*} \widehat{p}+\widehat{\eta}
\end{aligned}
$$

with some $\widehat{p} \in C(\bar{x}), \widehat{\eta} \in(C(\bar{x}))^{\circ}$.

The rest follows from Lemmas 3.1, 3.2.

The above conditions mimic the concept of strong stationarity introduced in [15] for finite-dimensional MPECs. Hence, optimality conditions of Theorem 3.3 are sharper than the conditions in [8], based on the M(ordukhovich)-stationarity.

Example 1. Consider the MPEC from [8] defined by $\Omega=(0,1)$,

$$
\varphi(x, y)=\langle g, x\rangle+\int_{1 / 4}^{3 / 4} y(s) d s
$$

$\mathscr{A} y=-\triangle y$, and

$$
a(s)=\left\{\begin{aligned}
-2 & \text { for } s \in\left[0, \frac{1}{4}\right] \cup\left[\frac{3}{4}, 1\right] \\
0 & \text { otherwise }
\end{aligned}\right.
$$


In $(3.6)$

$$
g: s \mapsto \begin{cases}0 & \text { for } s \in\left[0, \frac{1}{4}\right) \cup\left(\frac{3}{4}, 1\right] \\ -\frac{1}{16}\left(s-\frac{1}{4}\right) & \text { for } s \in\left[\frac{1}{4}, \frac{1}{2}\right] \\ -\frac{1}{64}+\frac{1}{16}\left(s-\frac{1}{2}\right) & \text { for } s \in\left(\frac{1}{2}, \frac{3}{4}\right] .\end{cases}
$$

It is easy to see that the pair

$$
\begin{aligned}
\widehat{x} & =-\delta_{1 / 4}-\delta_{3 / 4} \\
\widehat{y}(s) & = \begin{cases}-s^{2}+\frac{1}{4} s & \text { on }\left[0, \frac{1}{4}\right] \\
-s^{2}+\frac{7}{4} s-\frac{3}{4} & \text { on }\left[\frac{3}{4}, 1\right] \\
0 & \text { otherwise }\end{cases}
\end{aligned}
$$

is a (local) solution of this MPEC. The optimality conditions (3.5) of Theorem 3.3 attain the form

$$
\begin{aligned}
& 0=g+\widehat{p} \\
& 0=\gamma+\widehat{\eta},
\end{aligned}
$$

where $\gamma$ is the characteristic function of the interval $[1 / 4,3 / 4]$. Since $\bar{\mu}=\bar{x}$, one has

$$
\begin{aligned}
& L(\widehat{y})=\left(0, \frac{1}{4}\right) \cup\left(\frac{3}{4}, 1\right), \\
& I_{+}(\widehat{\mu})=\left\{\frac{1}{4}\right\} \cup\left\{\frac{3}{4}\right\},
\end{aligned}
$$

and we observe that the multipliers $(\widehat{p}, \widehat{\eta})=(-g,-\gamma)$ fulfill all conditions (i) - (iv) of Theorem 3.3.

\section{Constrained case}

The situation changes, however, whenever we have to do with control or state constraints.

The following approach relies on the local fuzzy sum rule due to A.D. Ioffe ([3], [5]). Observe first that a (local) solution $(\widehat{x}, \widehat{y})$ of $(2.1)$ is a minimizer of the sum

$$
\varphi(x, y)+\delta_{\mathrm{Gr} S}(x, y)+\delta_{\omega \times \Xi}(x, y)
$$

over a neighborhood $\mathscr{O}$ of $(\widehat{x}, \widehat{y})$. In what follows we employ the powerful notion of calmness as a qualification condition.

Definition 4.1. A multifunction $\Phi$ between Banach spaces $U$ and $V$ is said to be calm at a point $(\bar{u}, \bar{v}) \in \operatorname{Gr} \Phi$, provided there exist a nonnegative modulus $L$ and neighborhoods $\mathscr{U}$ of $\bar{u}$ and $\mathscr{V}$ of $\bar{v}$ such that

$$
\Phi(u) \cap \mathscr{V} \subset \Phi(\bar{u})+L\|u-\bar{u}\| \mathbb{B} \text { for all } u \in \mathscr{U} .
$$

Lemma 4.2. Assume that the "perturbation" map $M\left[H^{-1}(\Omega) \times \stackrel{\circ}{H}^{1}(\Omega) \rightrightarrows H^{-1}(\Omega) \times \stackrel{\circ}{H}^{1}(\Omega)\right]$ defined by

$$
M\left(q_{1}, q_{2}\right)=\left\{(x, y) \in \operatorname{Gr} S ; x-q_{1} \in \omega, y-q_{2} \in \Xi\right\}
$$

is calm at $(0,0, \widehat{x}, \widehat{y})$. Then there is a closed ball $B$ centered at $(\widehat{x}, \widehat{y})$ such that the inequality

$$
\begin{aligned}
& \varphi(\widehat{x}, \widehat{y}) \leq \liminf _{\nu \searrow 0}\left\{\varphi\left(x_{1}, y_{1}\right) ;\left(x_{1}, y_{1}\right) \in B, \exists \text { points }\left(x_{2}, y_{2}\right),\left(x_{3}, y_{3}\right) \in B\right. \\
& \text { such that } \left.y_{2}=S\left(x_{2}\right), x_{3} \in \omega, y_{3} \in \Xi \text { and } \operatorname{diam}\left\{\left(x_{1}, y_{1}\right),\left(x_{2}, y_{2}\right),\left(x_{3}, y_{3}\right)\right\} \leq \nu\right\},
\end{aligned}
$$

holds true. 
Proof. Let $\mathscr{V}$ be a neighborhood of $(\widehat{x}, \widehat{y})$ from the definition of calmness and $L \geq 0$ be the respective modulus. We can definitely shrink $B$ if necessary to achieve $B \subset \mathscr{V}$ and $2 B \subset \mathscr{O}$. Let $l$ be a Lipschitzian modulus of $\varphi$ on $\mathscr{O}$ and let $V$ stand for the quantity on the right-hand side of (4.2). Clearly, since $\left\|\left(x_{1}, y_{1}\right)-\left(x_{2}, S\left(x_{2}\right)\right)\right\| \leq \nu, V$ admits the lower bound

$$
\begin{gathered}
V \geq \liminf _{\nu \searrow 0}\left\{\varphi\left(x_{2}, S\left(x_{2}\right)\right)-l \nu ;\left(x_{2}, S\left(x_{2}\right)\right) \in B, \exists\left(x_{3}, y_{3}\right) \in B\right. \text { such that } \\
\left.x_{3} \in \omega, y_{3} \in \Xi,\left\|\left(x_{2}, S\left(x_{2}\right)\right)-\left(x_{3}, y_{3}\right)\right\| \leq \nu\right\} .
\end{gathered}
$$

The last inequality at the right hand side of (4.3) implies that

$$
\left(x_{2}, S\left(x_{2}\right)\right) \in M\left(q_{1}, q_{2}\right)
$$

with $q_{1}=x_{2}-x_{3}, q_{2}=S\left(x_{2}\right)-y_{3}$. Since $\left(x_{2}, S\left(x_{2}\right)\right) \in B$, by the calmness of $M$ to each $\nu$ sufficiently small there is a point $(\widetilde{x}, \widetilde{y}) \in M(0,0)$ (i.e. $\widetilde{y}=S(\widetilde{x})$ with $\widetilde{x} \in \omega$ and $\widetilde{y} \in \Xi$ ) such that

$$
\left\|\left(x_{2}, S\left(x_{2}\right)\right)-(\widetilde{x}, \widetilde{y})\right\| \leq(L+1) \nu .
$$

Hence it follows from (4.3) and the inclusion $2 B \subset \mathscr{O}$ that for $\nu$ sufficiently small

$$
V \geq \liminf _{\nu \backslash 0}\{\varphi(\widetilde{x}, \widetilde{y})-l \nu-l(L+1) \nu ; \widetilde{y}=S(\widetilde{x}), \widetilde{x} \in \omega, \widetilde{y} \in \Xi,(\widetilde{x}, \widetilde{y}) \in \mathscr{O}\}
$$

Since $(\widehat{x}, \widehat{y})$ is a local minimum of $\varphi$ on $\mathscr{O} \cap \operatorname{Gr} S \cap \omega \times \Xi$, the limes inferior above amounts to $\varphi(\widehat{x}, \widehat{y})$ and we are done.

On the basis of Lemma 3.4 we can now derive the following fuzzy optimality conditions for $(2.1)$.

Theorem 4.3. Let $(\widehat{x}, \widehat{y})$ be a (local) solution of (2.1)) and assume that the mapping (4.1) is calm at $(0,0, \widehat{x}, \widehat{y})$. Then, for any $\varepsilon>0$, there exist points $\left(x_{1}, y_{1}\right),\left(x_{2}, y_{2}\right),\left(x_{3}, y_{3}\right) \in$ $(\widehat{x}, \widehat{y})+\varepsilon \mathbb{B}$ with $y_{2}=S\left(x_{2}\right), x_{3} \in \omega, y_{3} \in \Xi$ and points $p \in \stackrel{\circ}{H}^{1}(\Omega), \eta \in H^{-1}(\Omega), \gamma \in$ $\stackrel{\circ}{H}^{1}(\Omega), \psi \in H^{-1}(\Omega)$ such that, with $\mu_{2}=-\mathscr{A} y_{2}+x_{2}-a$, one has

$$
\begin{aligned}
& p \geq 0 \text { q.e. on } I_{0}\left(y_{2}, \mu_{2}\right) \\
& p=0 \mu_{2} \text {-a.e.on } I_{+}\left(\mu_{2}\right) \\
& \langle\eta, z\rangle=0 \text { for all } z \in \stackrel{\circ}{H}^{1}(\Omega) \text { such that } z=0 \text { q.e. on } I\left(y_{2}\right) ; \\
& \langle\eta, z\rangle \leq 0 \text { for all } z \in D \text { such that }\left\langle\mu_{2}, z\right\rangle=0 \\
& \gamma \in \widehat{N}_{\omega}\left(x_{3}\right) \\
& \psi \in \widehat{N}_{\Xi}\left(y_{3}\right) \text {. }
\end{aligned}
$$

Moreover,

$$
\left|\varphi\left(x_{1}, y_{1}\right)-\varphi(\widehat{x}, \widehat{y})\right|<\varepsilon
$$

and

$$
\left\|\left(\begin{array}{l}
\nabla_{x} \varphi\left(x_{1}, y_{1}\right)+p+\gamma \\
\nabla_{y} \varphi\left(x_{1}, y_{1}\right)-\mathscr{A}^{*} p+\eta+\psi
\end{array}\right)\right\|<\varepsilon .
$$

Proof. The calmness of $M$ ensures by virtue of Lemma 4.1 the so-called local uniform lower semicontinuity of the system $\left(\varphi, \delta_{\operatorname{Gr} S}, \delta_{\omega \times \Xi}\right)$ at $(\widehat{x}, \widehat{y})$, cf. [3, Def.2.4]. The rest follows from [3, Theorem 2.6] due to Lemmas 3.1, 3.2.

It is easy to show that the calmness condition of Theorem 4.2 is automatically fulfilled, whenever one has to do with control constraints only. 
Proposition 4.4. Let $\Xi=\stackrel{\circ}{H}^{1}(\Omega)$. Then $M$ is calm at any feasible pair $(\bar{x}, \bar{y})$.

Proof. Let us endow the carthesian product of the control and the state space with the sum norm. Take any $(x, y) \in M\left(q_{1}, q_{2}\right)$ for an arbitrary $\left(q_{1}, q_{2}\right)$ close to 0 , and put $\widetilde{x}=x-q_{1} \in \omega$. Clearly,

$$
d_{M(0,0)}(x, y) \leq\|x-\widetilde{x}\|+\|S(x)-S(\widetilde{x})\| \leq(l+1)\left\|q_{1}\right\|,
$$

where $l$ is the Lipschitz modulus of $S$, and we are done.

In presence of state constraints one can sometimes make use of the following statement which is, similarly to Lemma 4.2 and Proposition 4.3, relevant for a general class of MPECs with locally Lipschitz $S$.

Proposition 4.5. $M$ is calm at $(0,0, \bar{x}, \bar{y})$ if and only if the multifunction $\widetilde{M}\left[\stackrel{\circ}{H}^{1}(\Omega) \rightarrow\right.$ $\left.H^{-1}(\Omega)\right]$, given by

$$
\widetilde{M}(q):=\{x \in \omega ; S(x)-q \in \Xi\}
$$

is calm at $(0, \bar{x})$.

Proof. Clearly, one has that

$$
M\left(q_{1}, q_{2}\right)=\left\{(x, S(x)) ; x \in M_{1}\left(q_{1}, q_{2}\right)\right\}
$$

where

$$
M_{1}:\left(q_{1}, q_{2}\right) \mapsto\left\{x ; x-q_{1} \in \omega, S(x)-q_{2} \in \Xi\right\} .
$$

Since $S$ is single-valued and Lipschitz, the calmness of $M$ at $(0,0, \bar{x}, \bar{y})$ is equivalent to the calmness of $M_{1}$ at $(0,0, \bar{x})$. Further, it is clear that the calmness of $M_{1}$ at $(0,0, \bar{x})$ implies the calmness of $\widetilde{M}$ at $(0, \bar{x})$, and so it suffices to prove the reverse implication.

Assume by contradiction the existence of sequences

$$
x^{(i)} \rightarrow \bar{x},\left(q_{1}^{(i)}, q_{2}^{(i)}\right) \rightarrow(0,0) \text { with } x^{(i)} \in M_{1}\left(q_{1}^{(i)}, q_{2}^{(i)}\right)
$$

such that

$$
d_{M_{1}(0,0)}\left(x^{(i)}\right) \geq i\left(\left\|q_{1}^{(i)}\right\|+\left\|q_{2}^{(i)}\right\|\right) \forall i .
$$

Put $\widetilde{x}^{(i)}:=x^{(i)}-q_{1}^{(i)}$ and observe that, due to

$$
S\left(x^{(i)}\right)-S\left(\widetilde{x}^{(i)}\right)+S\left(\widetilde{x}^{(i)}\right)-q_{2}^{(i)} \in \Xi
$$

one has $S\left(\widetilde{x}^{(i)}\right)-q^{(i)} \in \Xi$ with $q^{(i)}=S\left(\widetilde{x}^{(i)}\right)-S\left(x^{(i)}\right)+q_{2}^{(i)}$. By the Lipschitz continuity of $S$

$$
\left\|q^{(i)}\right\| \leq l\left\|\widetilde{x}^{(i)}-x^{(i)}\right\|+\left\|q_{2}^{(i)}\right\|=l\left\|q_{1}^{(i)}\right\|+\left\|q_{2}^{(i)}\right\| \leq \max \{l, 1\}\left(\left\|q_{1}^{(i)}\right\|+\left\|q_{2}^{(i)}\right\|\right),
$$

where $l$ is the Lipschitz constant of $S$. It follows that

$$
d_{\widetilde{M}(0)}\left(\widetilde{x}^{(i)}\right) \geq d_{M_{1}(0,0)}\left(x^{(i)}\right)-\left\|q_{1}^{(i)}\right\| \geq(i-1)\left(\left\|q_{1}^{(i)}\right\|+\left\|q_{2}^{(i)}\right\|\right) \geq \frac{i-1}{\max \{l, 1\}}\left\|q^{(i)}\right\|
$$

whence contradiction with the calmness of $\widetilde{M}$ at $(0, \bar{x})$. The result has been established. 
For testing of calmness of infinite-dimensional multifunctions we refer to [13]. The next step on our way to the $M$-stationarity conditions for (2.1) consists in the boundedness result below. Before we state it, let us introduce the following important notion ([12, Def. 1.67 (ii)]).

We say that a multifunction $F$ between Asplund spaces $U$ and $V$ is partially sequentially normally compact $(P S N C)$ at $(\bar{u}, \bar{v}) \in \operatorname{Gr} F$, if for any sequences $\left(u_{k}, v_{k}, u_{k}^{*}, v_{k}^{*}\right)$ satisfying

$$
\left(u_{k}, v_{k}\right) \stackrel{\operatorname{Gr} F}{\longrightarrow}(\bar{u}, \bar{v}), u_{k}^{*} \in \widehat{D}^{*} F\left(u_{k}, v_{k}\right)\left(v_{k}^{*}\right), u_{k}^{*} \stackrel{*}{\rightarrow} 0, v_{k}^{*} \rightarrow 0
$$

one has $u_{k}^{*} \rightarrow 0$. It is proved in [12, Proposition 1.68] that $F$ is PSNC at $(\bar{u}, \bar{v})$, if it has the Aubin property around $\bar{u}, \bar{v}$.

Lemma 4.6. Assume that $\Xi=\stackrel{\circ}{H}^{1}(\Omega)$ and consider a sequence $\varepsilon_{i} \downarrow 0$, and the corresponding sequences $x_{1}^{(i)}, y_{1}^{(i)}, x_{2}^{(i)}, y_{2}^{(i)}, x_{3}^{(i)}, y_{3}^{(i)}, p^{(i)}, \eta^{(i)}, \gamma^{(i)}$ generated by Theorem 4.2. Then, among the sequences of multipliers $\left\{\left(p^{(i)}, \eta^{(i)}\right)\right\}$ and Fréchet normals $\left\{\gamma^{(i)}\right\}$ there is at least one, say $\left\{\bar{p}^{(i)}, \bar{\eta}^{(i)}, \bar{\gamma}^{(i)}\right\}$, which is bounded.

Proof. Observe first that, as a contradiction to the above statement, it suffices to assume that one always has $\left\|p^{(i)}\right\| \rightarrow \infty$. Indeed, if $\left\{p^{(i)}\right\}$ is bounded, then necessarily, both corresponding sequences $\left\{\eta^{(i)}\right\}$ and $\left\{\gamma^{(i)}\right\}$ must be bounded as well by virtue of (4.5). So let us assume that for all considered sequences $x_{1}^{(i)}, y_{1}^{(i)}, x_{2}^{(i)}, y_{2}^{(i)}, x_{3}^{(i)}, y_{3}^{(i)}, p^{(i)}, \eta^{(i)}, \gamma^{(i)}$ it holds that $\left\|p^{(i)}\right\| \rightarrow \infty$.

By the local fuzzy sum rule there is at least one sequence, say $\left\{\left(\bar{x}_{1}^{(i)}, \bar{y}_{1}^{(i)}, \bar{x}_{2}^{(i)}, \bar{y}_{2}^{(i)}, \bar{x}_{3}^{(i)}, \bar{y}_{3}^{(i)}, c^{(i)}, d^{(i)}\right)\right\} \quad$ such that $c^{(i)} \in \widehat{N}_{\mathrm{Gr} S}\left(\bar{x}_{2}^{(i)}, \bar{y}_{2}^{(i)}\right) \quad$ and $d^{(i)} \in \widehat{N}_{\omega \times H^{1}(\Omega)}\left(\bar{x}_{3}^{(i)}, \bar{y}_{3}^{(i)}\right)$, and

$$
\left\|\nabla \varphi\left(\bar{x}_{1}^{(i)}, \bar{y}_{1}^{(i)}\right)+c^{(i)}+d^{(i)}\right\|<\varepsilon_{i} .
$$

It follows from the proof of Theorem 3.4 that for all $i=1,2, \ldots$ the elements $c^{(i)}$ admit the representation $\left(\bar{p}^{(i)},-\mathscr{A}^{*} \bar{p}^{(i)}+\bar{\eta}^{(i)}\right)$ and $d^{(i)}=\left(\bar{\gamma}^{(i)}, 0\right)$ with $\bar{\gamma}^{(i)} \in \widehat{N}_{\omega}\left(\bar{x}_{3}^{(i)}\right)$. Consequently, it holds that

$$
\left\|\frac{\nabla_{y} \varphi\left(\bar{x}_{1}^{(i)}, \bar{y}_{1}^{(i)}\right)}{\left\|\bar{p}^{(i)}\right\|}-\mathscr{A}^{*} \frac{\bar{p}^{(i)}}{\left\|\bar{p}^{(i)}\right\|}+\frac{\bar{\eta}^{(i)}}{\left\|\bar{p}^{(i)}\right\|}\right\| \downarrow 0 .
$$

Clearly, for all $i$,

$$
\left(\frac{\bar{p}^{(i)}}{\left\|\bar{p}^{(i)}\right\|},-\mathscr{A}^{*} \frac{\bar{p}^{(i)}}{\left\|\bar{p}^{(i)}\right\|}+\frac{\bar{\eta}^{(i)}}{\left\|\bar{p}^{(i)}\right\|}\right) \in \widehat{N}_{\mathrm{Gr} S}\left(\bar{x}_{2}^{(i)}, \bar{y}_{2}^{(i)}\right),
$$

and

$$
-\mathscr{A}^{*} \frac{\bar{p}^{(i)}}{\left\|\bar{p}^{(i)}\right\|}+\frac{\bar{\eta}^{(i)}}{\left\|\bar{p}^{(i)}\right\|} \rightarrow 0
$$

by virtue of (4.6). By the PSNC property of $S$ at $(\widehat{x}, \widehat{y})$, all weakly convergent subsequences of the sequence of unit vectors $\left\|\bar{p}^{(i)}\right\|^{-1} \bar{p}^{(i)}$ must converge to nonzero vectors. Let $a$ be one of these accumulation points. Then one has

$$
a \in D^{*} S(\widehat{x}, \widehat{y})(0)
$$

whence a contradiction with the Lipschitz continuity of $S$. It follows that $\left\{\bar{p}_{(i)}\right\}$ is bounded and the proof is complete.

After this preparatory work we are now able to recover the main result of [8]. 
Theorem 4.7. Let $\Omega$ be the open interval $(0,1) \subset \mathbb{R}$ and $\mathscr{A}=-\triangle$. Further assume that $(\widehat{x}, \widehat{y})$ is a (local) solution of the respective problem (2.1). Then there exist points $\widehat{p} \in$ $\stackrel{\circ}{H}^{1}(\Omega), \widehat{\eta} \in H^{-1}(\Omega)$ such that

$$
\begin{aligned}
& 0 \in \nabla_{x} \varphi(\widehat{x}, \widehat{y})+\widehat{p}+N_{\omega}(\widehat{x}) \\
& 0=\nabla_{y} \varphi(\widehat{x}, \widehat{y})-\mathscr{A}^{*} \widehat{p}+\widehat{\eta}
\end{aligned}
$$

In addition, the multipliers $\widehat{p}, \widehat{\eta}$ fulfill the conditions

(i) $\langle\eta, h\rangle=0$ for all test functions $h \in \stackrel{\circ}{H}^{1}(0,1)$ such that $\operatorname{supp} h \subset L(\widehat{y})$;

(ii) $p(s)=0$ for $s \in I_{+}(\triangle \widehat{y}+\widehat{x}-a)$;

(iii) for each open interval $\mathscr{U} \subset[0,1]$ with $p(s)<0$ for $s \in \mathscr{U}$ one has

$$
\langle\eta, h\rangle=0
$$

for all test functions $h \in \stackrel{\circ}{H}^{1}(0,1)$ such that $\operatorname{supp} h \subset \overline{\mathscr{U}}$;

(iv) for each open interval $\mathscr{U} \subset[0,1]$ with $p(s)>0$ for $s \in \mathscr{U}$ one has

$$
\langle\eta, h\rangle \leq 0
$$

for all test functions $h \in D$ such that $\operatorname{supp} h \subset \overline{\mathscr{U}}$.

Proof. Put $\varepsilon=\varepsilon_{i} \downarrow 0$ in Theorem 4.2. In this way we obtain sequences $\left(x_{1}^{(i)}, y_{1}^{(i)}\right) \rightarrow$ $(\widehat{x}, \widehat{y}), \quad\left(\widehat{x}_{2}^{(i)}, \widehat{y}_{2}^{(i)}\right) \stackrel{\operatorname{Gr} S}{\longrightarrow}(\widehat{x}, \widehat{y}),\left(x_{3}^{(i)}, y_{3}^{(i)}\right) \longrightarrow(\widehat{x}, \widehat{y})$ with $x_{3}^{(i)} \in \omega, y_{3}^{(i)} \in \stackrel{\circ}{H}^{1}(\Omega),\left\{p^{(i)}\right\}$, $\left\{\eta^{(i)}\right\},\left\{\gamma^{(i)}\right\}$ satisfying all conditions of Theorem 4.2. By Lemma 4.6 the sequences $\left\{p^{(i)}\right\}$, $\left\{\eta^{(i)}\right\},\left\{\gamma^{(i)}\right\}$ possess weakly convergent subsequences. Our task is thus to find out conditions which relate $(\widehat{x}, \widehat{y})$ and the weak limits $\widehat{p}, \widehat{\eta}, \widehat{\gamma}$. It is easy to see that by definition certainly $\widehat{\gamma} \in N_{\omega}(\widehat{x})$. The first two of conditions (4.4) attain in fact the form

$$
\begin{aligned}
& p^{(i)} \geq 0 \text { on } I_{0}\left(y_{2}^{(i)}, \mu_{2}^{(i)}\right) \\
& p^{(i)}=0 \text { on } I_{+}\left(\mu_{2}^{(i)}\right),
\end{aligned}
$$

because the functions $p^{(i)}$ are continuous. The third condition in (4.4) clearly implies condition (10) and the fourth condition is identical with condition (11) in [8, Proposition 4]. Thus it suffices to apply Lemma 6 and Propositions 7-10 from [8] to arrive at above conditions (i)-(iv). These results from [8] rely on the compact embedding of $\stackrel{\circ}{H}^{1}(0,1)$ to $C^{0}(0,1)$ which is not valid for domains of higher dimension.

Condition (4.7) follows from (4.5) and we are done.

Remark 4.8. It is easy to see that in the case $\omega=H^{-1}(\Omega)$ the conditions of Theorem 4.7 are less sharp than the respective conditions of Theorem 3.4. 


\section{Two-dimensional case}

When proving the limiting optimality conditions in Theorem 4.7, we strongly used the compact embedding of $H^{1}(\Omega)$ to $C^{0}(\bar{\Omega})$ (endowed with the Chebyshev norm). It enabled us to pass from a weak convergence of a sequence $u^{(n)}$ to $u$ in $H^{1}(\Omega)$ to the uniform convergence of $u^{(n)}$ on $\bar{\Omega}$. In the case of an open domain $\Omega \subset \mathbb{R}^{d}$ with $d>1$ weak convergence of $u^{(n)}$ in $H^{1}(\Omega)$ implies strong convergence only in $L^{p}(\Omega)$ with $p<2 d /(d-2)$ for $d>2$ and in any $L^{p}(\Omega)$ for $d=2$. Thus a (not relabelled) subsequence converges almost everywhere and according to Jegorov's theorem for any $\epsilon>0$ there is a subset $M \subset \Omega$ with Lebesgue measure $\lambda(M)<\epsilon$ and $u^{(n)} \rightarrow u$ uniformly on $\Omega \backslash M$. However, estimates of Lebesgue measure of $M$ are too weak to work with measures in $H^{-1}(\Omega)$. We would need an analogous assertion with the estimates in terms of capacity instead of Lebesgue measure. Unfortunately, such an assertion does not hold, too. A counterexample was suggested by E. De Giorgi and proposed by J. Frehse for the dimension $d \geq 3$ in [6], [7]. It seems that an example in space dimension 2 was published in a research report without access to a copy. For this reason we present here another example which can be very similar to J. Frehse's one. In the example we construct a sequence $v^{(n)} \rightarrow 0$ in $H^{1}(\Omega)$ such that $\operatorname{cap}\left\{v^{(n)}=1\right\}$ is bounded away from zero.

\section{Step 1.}

Let $R>\rho$ be positive real numbers, $s \in R^{2}$. Denote by $|x|$ the Euclidean norm of $x$. Besides $\mathbb{B}(s, \rho)=\left\{x \in R^{2} ;|x-s|<\rho\right\}$, we define $\mathbb{S}(s, \rho)=\left\{x \in R^{2} ;|x-s|=\rho\right\}$ and $\mathbb{Q}(s, \rho)=\left\{x=\left[x_{1}, x_{2}\right] \in R^{2} ; \max \left\{\left|x_{1}-s_{1}\right|,\left|x_{2}-s_{2}\right|\right\} \leq \rho\right\}$.

In fact, infimum in the definition of capacity is attained when taking $u=1$ on $\mathbb{B}(s, \rho)$ and $u$ minimizing Dirichlet integral on $\mathbb{B}(s, R) \backslash \overline{\mathbb{B}}(s, \rho)$ with boundary values $u=1$ on $\mathbb{S}(s, \rho)$ and $u=0$ on $\mathbb{S}(s, R)$. The corresponding solution $u$ is radially symmetric with respect to $s$. For given $R, \rho \in \mathbb{R}^{+}, s \in \mathbb{R}^{2}$ consider

$$
u_{R, \rho, s}(x)= \begin{cases}1 & \text { on } \mathbb{B}(s, \rho), \\ \frac{\ln \frac{R}{|x-s|}}{\ln \frac{R}{\rho}} & \text { on } \mathbb{B}(s, R) \backslash \overline{\mathbb{B}}(s, \rho), \\ 0 & \text { on } \mathbb{R}^{2} \backslash \mathbb{B}(s, R) .\end{cases}
$$

Then $\int_{\mathbb{R}^{2}}|\nabla u|^{2}=\int_{\mathbb{B}(s, R)}|\nabla u|^{2}=2 \pi\left(\ln \frac{R}{\rho}\right)^{-1}$.

\section{Step 2.}

Assume that a compact set $K$ lies strictly inside the unit disc $\mathbb{B}$. Then it holds (see $[9$, Chapter II, paragraph 4.,p.168], )

$$
\operatorname{cap}(K)=\max \{\mu(1)\}
$$

where $\mu(1)=\int_{K} 1 d \mu(y)$ is the measure of the support of $\mu$ and maximum is taken over all nonnegative measures $\mu$ supported in $K$ for which

$$
U_{\mu}(x)=\int_{K} \ln \frac{1}{|x-y|} d \mu(y) \leq 1
$$

on $\mathbb{R}^{2}$. For $K=\mathbb{S}(s, \rho)$ and $\mu$ the measure obtained by the uniform distribution of a unit mass over $\mathbb{S}(s, \rho)$ we have 


$$
\begin{aligned}
& \mu(f)=\frac{1}{2 \pi \rho} \int_{\mathbb{S}(s, \rho)} f(x) d S, \\
& U_{\mu}(x)=\frac{1}{2 \pi \rho} \int_{\mathbb{S}(s, \rho)} \ln \frac{1}{|x-y|} d S(y),
\end{aligned}
$$

where the integrals over the sphere are the surface integrals of the first kind. In a rather cumbersome calculation of $U_{\mu}$, the differentiation in the parameter $a$ for the the necessary evaluation of the integral

$$
\int_{-\pi}^{\pi} \ln (1+a \cos \alpha) d \alpha
$$

helps. It holds

$$
U_{\mu}(x)= \begin{cases}\ln \frac{1}{|x-s|}, & \text { for }|x-s| \geq \rho \\ \ln \frac{1}{\rho}, & \text { for }|x-s|<\rho .\end{cases}
$$

\section{Step 3.}

Denote $\mathbf{0} \equiv[0,0], \mathbb{Q}=\mathbb{Q}(\mathbf{0}, 1 / 2) \subset \subset \mathbb{B}$. For even $n \in N, k, j \in N_{o}$ set $R=1 / 4 n ; s_{k, j}=$ $[k / 2 n, j / 2 n], \mathbb{B}_{k, j}=\mathbb{B}\left(s_{k, j}, 1 / 4 n\right)$ for $k, j \in\{-n, \ldots, n\}$. Positive $\rho \in(0, R)$ is defined in (5.1) below. We simplify the notation by writing $u_{n, k, j}$ instead of $u_{R, \rho, s}$ for above described $R, \rho, s$. Define $v^{(n)}=\sum_{k, j=-n}^{n} u_{n, k, j}$.

Then for a (not relabelled) subsequence it holds

1. $\left(v^{(n)}\right)$ is bounded in $H^{1}(\mathbb{B})$ and in $L_{\infty}(\mathbb{B})$,

2. $v^{(n)} \rightarrow 0$ in $H^{1}(\mathbb{B})$,

3. There is an $\epsilon>0$ such that the capacity of

$$
\left\{x \in \mathbb{B} ; v^{(n)}(x)>\epsilon\right\}=\cup_{j, k \in\{-n, \ldots, n\}} \mathbb{B}\left(s_{k, j}, R^{1-\epsilon} \rho^{\epsilon}\right)
$$

is bounded from below by a positive constant that does not depend on $n$.

\section{Proof:}

1. It is obvious that $\left\|v^{(n)}\right\|_{L_{\infty}(\mathbb{B})}=1,\left\|v^{(n)}\right\|_{L_{2}(\mathbb{B})} \leq 1$. Moreover,

$$
\nabla u_{n, k, j}(x)= \begin{cases}0 & \text { on } \mathbb{B}\left(s_{k, j}, \rho\right) \cup\left(\mathbb{R}^{2} \backslash \overline{\mathbb{B}}\left(s_{k, j}, R\right)\right) \\ -\frac{2 \pi}{\ln \frac{R}{\rho}} \frac{x-s_{k, j}}{\left|x-s_{k, j}\right|^{2}} & \text { on } \mathbb{B}\left(s_{k, j}, R\right) \backslash \overline{\mathbb{B}}\left(s_{k, j}, \rho\right),\end{cases}
$$

and thus

$$
\left\|\nabla v^{(n)}\right\|_{L_{2}(\mathbb{B})}=2 \pi \frac{(n+1)^{2}}{\ln \frac{R}{\rho}}=2 \pi
$$

for

$$
\ln \frac{R}{\rho}=(n+1)^{2}, \text { i.e. for } \rho=\frac{1}{4 n} e^{-(n+1)^{2}} .
$$

2. To get the weak convergence of gradients of $v^{(n)}$ to zero in $L_{2}(\mathbb{B})$ it is enough to show that

$$
\int_{\mathbb{B}} \frac{\partial v^{(n)}}{\partial x_{i}} \psi d x \rightarrow 0
$$


for $i=1,2$ and $\psi$ a characteristic function of a rectangle $[a, b] \times[c, d] \subset \mathbb{B}$ (because linear hull of such functions is dense in $\left.L_{2}(\mathbb{B})\right)$. In case $\mathbb{B}_{j k} \subset[a, b] \times[c, d]$ it holds

$$
\int_{\mathbb{B}_{j k}} \frac{\partial u_{n, k, j}}{\partial x_{i}} d x=0
$$

as $u_{n, k, j}=0$ on $\partial \mathbb{B}_{j k}$. In case $\mathbb{B}_{j k} \cap \partial([a, b] \times[c, d]) \neq \emptyset$ we have

$$
\int_{\mathbb{B}_{j k} \cap([a, b] \times[c, d])}\left|\nabla u_{n, k, j}\right| \leq\left(2 \pi^{2} R\left(\ln \frac{R}{\rho}\right)^{-1}\right)^{1 / 2} .
$$

As there are only $2(2 n+1)(b-a+d-c)$ balls $\mathbb{B}_{j, k}$ with this property we get that

$$
\int_{\mathbb{B}}\left|\nabla v^{(n)}(x)\right| \psi(x) d x \leq 2(2 n+1)(b-a+d-c) 2 \pi^{2}\left(4 n \ln \frac{R}{\rho}\right)^{-1} \rightarrow 0
$$

3. Denote $m_{n}=\frac{1}{(n+1)^{2}}$,

$$
U_{j k}^{(n)}(x)=\frac{m_{n}}{2 \pi \rho} \int_{\mathbb{S}\left(s_{j k}, \rho\right)} \ln \frac{1}{|x-y|} d S(y),
$$

i.e.

$$
U_{j k}^{(n)}(x)= \begin{cases}m_{n} \ln \frac{1}{\rho} & \text { for }\left|x-s_{j k}\right|<\rho \\ m_{n} \ln \frac{1}{\left|x-s_{j k}\right|} & \text { for }\left|x-s_{j k}\right| \geq \rho,\end{cases}
$$

the potential corresponding to the measure $\mu_{n}$ obtained by the uniform distribution of a mass $m_{n}$ over the sphere $\mathbb{S}\left(s_{j k}, \rho\right)$ and, finally, $U^{(n)}(x)=\sum_{j, k=-n}^{n} U_{j k}^{(n)}(x)$. and

Then for all $x \in \mathbb{Q}\left(\left[j_{0}, k_{0}\right], R\right)$ and $s_{j k} \neq s_{j_{0} k_{0}}$ we have $\left|x-s_{j k}\right| \geq \frac{1}{4 n} \max \left\{\left|j-j_{0}\right|,\left|k-k_{0}\right|\right\}$

$$
\begin{aligned}
U^{(n)}(x) & \leq m_{n}\left(\ln \frac{1}{\rho}+\sum_{\max \left(\left|j-j_{0}\right|,\left|k-k_{0}\right|\right)=1}^{n} \ln \frac{1}{\left|x-s_{j k}\right|}\right) \\
& \leq m_{n}\left(\ln \frac{1}{\rho}+\sum_{L=1}^{n}\left(\sum_{\max \left(\left|j-j_{0}\right|,\left|k-k_{0}\right|\right)=L} \ln \frac{4 n}{\max \left(\left|j-j_{0}\right|,\left|k-k_{0}\right|\right.}\right)\right) \\
& \leq m_{n}\left(\ln \frac{1}{\rho}+\sum_{L=1}^{n} 8 L \ln 4 n / L\right) .
\end{aligned}
$$

Moreover, the monotonicity of $f(x)=x \cdot \ln x$ on $[1, \infty)$ implies that

$$
\sum_{L=1}^{n+1} L \ln L \geq \int_{1}^{n} x \ln x d x \geq \frac{n^{2}}{2}(\ln n-1 / 2)
$$

hence

$$
U^{(n)}(x) \leq a_{n} \equiv\left(1+\frac{\ln (4 n)}{(n+1)^{2}}+8\left(\frac{n \ln 4 n}{2(n+1)}-\frac{n^{2}}{2(n+1)^{2}}(\ln n-1 / 2)-\frac{\ln (n+1)}{(n+1)}\right)\right) .
$$

As the squares $\mathbb{Q}\left(\left[j_{0}, k_{0}\right], R\right)$ cover $\mathbb{Q}$ the estimate holds on $\mathbb{Q}$ and it is easy to realize that it holds on $\mathbb{R}^{2}$. Denote by $B$ the upper bound of the (bounded) sequence $a_{n}$. Then $\frac{1}{B}(n)$ is an admissible potential in the definition of capacity of the set $K=\cup_{k, j \in\{-n, \ldots, n\}} \mathbb{B}_{k, j}$ and $\operatorname{cap}(K) \geq \frac{1}{B}>0$ for the positive constant $B$ that does not depend on $n$. 


\section{Conclusion}

The counterexample of the preceding section strikingly shows the importance of the compact imbedding of the used state space in $C^{0}(\bar{\Omega})$ in the derivation of limiting optimality conditions. So, to establish such conditions, it will be essential to find a different function-space setting for (2.1). Let $X$ and $Y$ be a control and state space, respectively, satisfying the following requirements:

1. $Y$ is compactly imbedded into $C^{0}(\bar{\Omega})$;

2. the elements of $N_{D}$ are signed Radon measures.

In this way we may loose other two important properties: the directional differentiability of $S$ and the surjectivity of the linear mapping from $X \times Y$ to $Y \times Y^{*}$, defined by

$$
\left(\begin{array}{ll}
0 & \mathrm{Id} \\
\mathscr{I}_{Y^{*}} & \mathscr{A}
\end{array}\right)
$$

where $\mathscr{I}_{Y^{*}}$ means the canonical injection of $X$ into $Y^{*}$. Observe that in the setting of [8] and this paper $X=Y^{*}$ so that the operator $\mathscr{I}_{Y^{*}}$ is indeed surjective. The possible lack of directional differentiability can be overcome by the technique from [8] and also the surjectivity is not indispensable, cf. [12], [13]. So we will try to follow this way in our next research.

\section{References}

[1] M. S. Bazaraa and C.M. Shetty (1979) Nonlinear Programming: Theory and Algorithms, Wiley, New York.

[2] J.F. Bonnans and A. Shapiro (2000), Perturbation Analysis of Optimization Problems, Springer, New York.

[3] J.M. Borwein and Q.J. Zhu (1999), A survey of subdifferential calculus with applications, Nonlinear Anal. 38, 687-773.

[4] A. Haraux (1975), How to differentiate the projection on a convex set in Hilbert space. Some application to variational inequalities, J. Math. Soc. Japan 29, 615-631.

[5] A.D. Ioffe (1984), Calculus of Dini subdifferentials of functions and contigent derivatives of set-valued maps, Nonlinear Anal. 8, 517-539.

[6] J. Frehse (1988), A refinement of Rellich's theorem Rend. Mat. 7 no. 3-4, 229-242 .

[7] J. Frehse (1982), Capacity methods in the theory of partial differential equations Jber.d.Dt. Math.Verein 84, 1-44.

[8] J. Jarušek and J.V. Outrata (2007), On sharp necessary optimality conditions in control of contact problems with strings, Nonlinear Anal. T.M.A. 67, 1117-1128.

[9] N. S. Landkof (1972),Foundations of Modern Potential Theory, Springer, Berlin-Heidelberg-New York.

[10] Z.-Q.Luo, J.S. Pang and D. Ralph (1996), Mathematical Programs with Equilibrium Constraints, Cambridge University Press, Cambridge.

[11] F. Mignot (1976), Contrôle dans les inéquations variationnelles elliptiques, J. Functional Anal. 22. 130-185. 
[12] B.S. Mordukhovich (2006), Variational Analysis and Generalized Differentiation I, Springer, New York.

[13] J.-P. Penot (2008), Error bounds, calmness and their applications in nonsmooth analysis. Preprint.

[14] R.T. Rockafellar and R. Wets (1998), Variational Analysis, Springer Verlag, Berlin.

[15] H. Scheel and S. Scholtes (2000), Mathematical programs with equilibrium constraints: Stationarity, optimality and sensitivity, Math. Oper. Res. 25, 1-22. 


\section{ACADEMY OF SCIENCES OF THE CZECH REPUBLIC MATHEMATICAL INSTITUTE}

The preprint series was founded in 1982. Its purpose is to present manuscripts of submitted or unpublished papers and reports of members of the Mathematical Institute of the Academy of Sciences of the Czech Republic.

The authors are fully responsible for the content of the preprints.

Mail address: $\quad$ Mathematical Institute,

Academy of Sciences of the Czech Republic

Žitná 25,

CZ-115 67 Praha 1

Czech Republic

phone: +420222090711

fax: +420222211638

e-mail: mathinst@math.cas.cz

http: //www.math.cas.cz

\section{Latest preprints of the series:}

\section{8}

182 Amiran Gogatishvili, Júlio S. Neves, Bohumír Opic: Optimal embeddings of Bessel-potential-type spaces into generalized Hölder spaces involving $k$-modulus of smoothness

181 Ivan Straškraba, Emil Vitásek: The flow of a liquid with cavitation

180 Bernard Ducomet, Šárka Nečasová: A boundary value problem for the spherically symmetric motion of a pressureless gas with a temperature-dependent viscosity

179 Bernard Ducomet, Šárka Nečasová: Free boundary problem for the equations of spherically symmetric motion of compressible gas with density-dependent viscosity

178 Amiran Gogatishvili, Júlio S. Neves, Bohumir Opic: Sharp estimates of the $k$-modulus of smoothness of Bessel potentials

177 António M. Caetano, Amiran Gogatishvili and Bohumír Opic: Sharp embeddings of Besov spaces involving only slowly varying smoothness

176 Christof Eck, Jiř́ Jarušek: Solvability of static contact problems with Coulomb friction for orthotropic material

\section{7}

175 I. Bock and J. Jarušek: Solvability of dynamic contact problems for elastic von Kármán plates

174 S. Kračmar, $\breve{S}$. Nečasová, and P. Penel: Anisotropic $L^{2}$-estimates of weak solutions to the stationary Oseen-type equations in 3D-exterior domain for a rotating body

173 B. Goldys and B. Maslowski: The Ornstein Uhlenbeck bridge and applications to Markov semigroups

172 Bohdan Maslowski and Jan Pospísil: Ergodicity and parameter estimates for infinite-dimensional fractional Ornstein-Uhlenbeck process

171 Zdeněk Halas and Milan Tvrdý: Singular Periodic Impulse Problems

170 M. Doubek, M. Markl and P. Zima: Deformation theory (Lecture notes)

169 Bohumir Opic: Embeddings of Bessel-potential-type spaces

168 António M. Caetano, Amiran Gogatishvili and Bohumír Opic: Sharp embeddings of Besov spaces involving only logarithmic smoothness

\section{6}

167 Alberto Cabada, Alexander Lomtatidze and Milan Tvrdý: Periodic problem with quasilinear differential operator and weak singularity

166 Eduard Feireisl and Šárka Nečasová: On the motion of several rigid bodies in a viscous multipolar fluid

165 S. Kračmar, $\breve{S}$. Nečasová, P. Penel: Anisotropic $L^{2}$-estimates of weak solutions to the stationary Oseen-type equations in $R^{3}$ for a rotating body

164 Patrick Penel and Ivan Straškraba: Construction of a Lyapunov functional for 1D-viscous compressible barotropic fluid equations admitting vacua 